doi: http://dx.doi.org/10.7124/bc.0009D2

\section{B-2. Characterization of the nuclear localization signal of the actin-binding Moesin protein}

Csaba Bajusz, Ildikó Kristó, Péter Borkúti, Zoltán Kovács, Péter Vilmos

Biological Research Centre of the Hung.Acad.Sci.

H-6726 Temesvári krt. 62. Szeged, Hungary

csaba.bajusz@gmail.com

The Moesin protein of Drosophila melanogaster is the single representative of the highly conserved Ezrin-Radixin-Moesin (ERM) protein family in the fly. ERM proteins play essential role in cytoskeletal rearrangements, since they act as a crosslinker between cell membrane proteins and the actin cytoskeleton. They all consist of an amino-terminal FERM and a carboxy-terminal actin-binding domain, and a flexible alpha helical region interconnecting the two terminal domains. The activity of ERMs is regulated via conformational change. In their inactive, closed arrangement the FERM and actin-binding domains interact with each other. The binding of Phosphatidylinositol 4,5-bisphosphate (PIP2) by the FERM domain, and subsequent phosphorylation of a Threonine residue at position 559 inhibits the intramolecular interaction, and as a result, the proteins acquire opened, active conformation. The cytosolic roles of ERM proteins are well characterized moreover, our laboratory demonstrated recently that in the nucleus Drosophila Moesin is involved in mRNA export. To gain deeper insight into the nuclear function of the protein, we decided to study its nuclear import in details. Methods: To achieve our goal, we generated DNA con- structs expressing mutant forms of Moesin tagged with GFP. The nuclear transport of the mutant proteins was monitored in cultured Drosophila cells where the import of Moesin was induced at the same time by the inhibition of mRNA export. Results: In the first set of experiments we determined the amino acids responsible for the nuclear localization (NLS). Next, the regulation of the NLS motif has been analyzed, and we found that the NLS is in fact bipartite and it is regulated neither by phosphorylation nor through monomeric actin binding. Finally, we determined if the protein enters the nucleus in its active or inactive conformation, monomeric or dimeric form, and in complex with G-actin. Conclusion: Our results reveal the fine details of the tightly regulated, active nuclear import of ERM proteins and enable the manipulation of their nuclear function.

doi: http://dx.doi.org/10.7124/bc.0009D3

\section{B-3. Deciphering the Role of Nuclear Lipid Islets in RNA Pol II transcription}

Can Balaban $^{1}$, Martin Sztacho ${ }^{1}$, Pavel Hozak ${ }^{1}$

Institute of Molecular Genetics ASCR v.v.i., Department of Biology of the Cell Nucleus, Vídeňská 1083, 142 20, Prague 4, Czech Republic can.balaban@img.cas.cz

Nuclear compartmentalization is crucial for spatiotemporal regulation of key processes such as transcription and splicing. Recently, we have identified an F-actin regulatory protein (MPRIP) in nucleus by using microscopy 
and biochemistry methods. Having two PH domains, MPRIP has an affinity to Phosphoinositols and as a key regulator of protein phosphatase 1 , it is a potential mediator for dephosphorylation of Pol II and splicing machinery (Mulder et al., 2003). Methods: We have first investigated the localization of the endogenous MPRIP by super-resolution microscopy (STED) and visualized that it forms nanoscale foci in the nucleoplasm, which partly coalesce with PIP2 containing structures in nucleus, particularly with nuclear speckles and sub-population of Nuclear Lipid Islets (NLIs) (Sobol et al., 2018). To study the dynamics of endogenous MPRIP nanoscale foci is extremely challenging, therefore we employ GFPMPRIP overexpression, in order to address question about the inner dynamics and mobility of MPRIP induced condensates. Results: The transient transfections experiments showed that highly expressing protein undergoes phase separation by accumulating in nucleus where it forms condensates that are up to $5 \mu \mathrm{m}$ in diameter. We presume that this formation is mediated by the $\mathrm{C}$ Terminal- Intrinsically Disordered Region (IDR). The Fluorescence recovery after photobleaching (FRAP) and live-cell imaging experiments uncovered their rapid motion, of which we have quantified the diffusion coefficients as a way to comprehend how the protein might act at endogenous levels. Conclusion: Here, we showed that MPRIP, an actin regulatory protein, is present in mammalian nucleus and partly localize to proximity of PIP2 rich structures. We hypothesize that MPRIP might regulate transcription by recruiting its known interactor, Protein phosphatase 1 , to the vicinity of NLIs. Altogether, these data indicates that MPRIP might be an impor- tant regulator of Pol II transcription, while the exact pathway remains to be enlightened.

This study was supported by the Czech Academy of Sciences (JSPS-18-18); Grant Agency of the Czech Republic (16-03346S, 17-09103S, 15-08738S).

References: Mulder, J., Poland, M., Gebbink, M.F.B.G., Calafat, J., Moolenaar, W.H., Kranenburg, O., 2003. p116Rip Is A Novel Filamentous Actin-binding Protein. J. Biol. Chem. 278, 27216-27223. Sobol, M., Hozak, P., 2018. Nuclear phosphatidylinositol 4,5-bisphosphate islets contribute to efficient RNA polymerase II-dependent transcription. Journal of Cell Science 131, jcs211094. https:// doi.org/10.1242/jcs.211094

doi: http://dx.doi.org/10.7124/bc.0009D5

\section{B-4. Abiotic stress effects - a model for studying the structure of plant nucleoloneme and chromatin transformations}

\author{
E. N. Baranova, L. I. Fedoreyeva, A. A. Gu- \\ levich \\ All-Russia Research Institute of Agricultural Bio- \\ technology, Timiriazevskaya 42, 127550 Moscow, \\ Russia \\ greenpro2007@rambler.ru
}

The study of ultrastructural transformations of the nuclear domains of cells of actively dividing tissues of the meristem under the influence of abiotic environmental factors such as cold, salinity, changes in the magnetic field parameters accompanying the change in the level of gene expression and chromatin modification allow visualizing these processes. We present the details of the process of structural organization, formation and disassembly of the nucleolus, chromatin condensation during the progression 